\title{
Immigration as a Social Determinant of Mental Health: Implications for Training and Education in Psychiatry
}

\author{
Poh Choo How ${ }^{1} \cdot$ Christine Kho $^{1} \cdot$ Raquel Rodríguez ${ }^{2} \cdot$ Ruth S. Shim $^{1}$ (D) \\ Received: 15 February 2020 / Accepted: 26 October 2020 / Published online: 9 January 2021 \\ (C) Academic Psychiatry 2021
}

The determinants of mental health are rooted in social, environmental, and economic conditions. These factors are primarily responsible for the health disparities and inequities seen across populations [1]. Discrimination, or the unjust treatment of different categories of people, is one of the most powerful social determinants of mental health, leading to increases in rates of depression, generalized anxiety disorder, post-traumatic stress disorder, alcohol use disorder, and mentally unhealthy days [2]. There are various types of discrimination in the United States (US). Discrimination based on gender, race, ethnicity, religion, immigration status, sexual orientation, gender identity, and others is on the rise [3]. As a result, this impact is felt by everyone within the healthcare system, including patients, medical students, residents, fellows, physicians, and other health care professionals and providers. Current immigration policy is closely tied to citizenship where citizens are able to sponsor the immigration of family members, with citizenship itself defined by changing definitions of "whiteness" and perpetuated by the fields of medicine and public health. [4].

Immigrants are less likely to utilize mental healthcare compared with US-born citizens, and children of undocumented immigrants have especially lower rates of receiving mental health services $[5,6]$. While these inequities have previously been attributed to issues of stigma and acculturation (i.e., lack of healthcare engagement is due to an individual's behavioral or cultural framework), immigration is increasingly being recognized as a social determinant of health [7]. Social determinants and structural competence perspectives look beyond the clinical

Ruth S. Shim

rshim@ucdavis.edu

University of California, Davis, Sacramento, CA, USA

University of California, San Francisco, San Francisco, CA, USA interaction to consider macrolevel sociopolitical, economical, and historical explanations for poor mental health outcomes.

It is vital that clinicians, educators, and leaders begin to work toward mental health equity for immigrant populations. In this paper, we discuss the concepts of cultural humility and structural competence as it relates to the psychiatry curriculum and clinical supervision of trainees working with immigrant populations. We also look through these lenses to help us understand the implications of changing immigration policies on immigrant trainees and on the psychiatric workforce as a whole.

\section{Cultural Humility and Structural Competence}

Cultural competence is a useful concept that emphasizes the need for providers to consider patients' cultural backgrounds and develop skills to effectively meet the cultural and linguistic needs of diverse patient populations, including immigrants. However, the term "competence" suggests that there is a default "normal" group who bear the responsibility of educating themselves on the "different" and by default "abnormal" group [8]. Furthermore, cultural competence implies that a finite limit of knowledge can be acquired to become an "expert" in describing the experiences of others [9]. This unintentionally promotes stereotyping, bias, and discrimination; contributes to two-dimensional and static views of patients; neglects consideration of intersectional identities; and risks harming patients from non-majority backgrounds. For example, a person cannot be defined merely by their race or ethnicity, but also their gender identity, sexual orientation, socioeconomic class, immigration status, and so on, that results in their facing overlapping and interdependent systems of discrimination and disadvantage.

In contrast, an informed understanding of the impact of immigration status on patients' mental health calls for a multidimensional, dynamic consideration of the patient in relation to the environmental, historical, sociopolitical, economical, and other structural contexts that contribute to the experiences 
of the patient. This view can be appreciated though the lens of cultural humility, a framework that (1) adopts a lifelong commitment to self-reflection and self-critique, (2) emphasizes the needs to address the power differential between patient and provider, and (3) develops mutually beneficial, nonpaternalistic clinical, community, and advocacy partnerships [10]. Cultural humility also necessitates an understanding of structural competence, which seeks to identify and address upstream policies and conditions that lead to healthcare inequities [11]. This calls for a paradigm shift in training of health professionals to learn how to elicit, diagnose/recognize, and address upstream sociopolitical influences on individual and population health. For example, recognizing that upstream policies excluding undocumented immigrants from access to free or affordable healthcare services negatively impacts their health, causing patients to wait until they are more severely ill before seeking care, rather than faulting the individual patient for having poor health practices.

\section{Implications for the Training of Residents and Fellows}

\section{Clinical Supervision}

In working with immigrants (whether documented/undocumented, established/newly arrived), a cultural humility approach requires that clinicians develop awareness of power dynamics and willingness to acknowledge their own limitations and inadequacies. This includes appreciating the added layer of power and privilege on the part of the provider, not only as a physician, but more frequently, as having the privilege of citizenship or other legal status, and the risks taken by the patient to seek help, especially if that patient is undocumented. Clinicians have an important opportunity in these encounters to legitimize any fear or mistrust of providers at both individual and organizational levels and to openly acknowledge their own deficiencies while inviting patients to partner with providers as experts of their own immigration experiences. It is important that faculty supervisors practice self-reflection in order to effectively model these behaviors to trainees. In the parallel of the supervisory relationship, in cases where the trainee has more experience/expertise either in cultural humility, structural competence, or in working with immigrant populations, increasing humility is called for on the part of the supervisor in deferring to the trainee's specific skills and knowledge, without tokenizing the trainee as a representative of a specific group. Of note, the lack of diversity among supervisors contributes to this inequity. Diversification of faculty is necessary to address this gap in knowledge and experience with regards to the immigration experience.

Operationalizing structural competence in the clinical encounter involves moving towards developing structural formulations of clinical cases by exploring systems that might be producing health inequities that affect the patient directly and indirectly [11]. For example, it would be important to elicit a patient's immigration status to understand if a patient is experiencing barriers in accessing healthcare, in order to refer patients to appropriate supportive resources (Table 1). At the same time, issues of unequal power/privilege and provider/system inadequacies complicate the process of eliciting a patient's immigration status. Direct solicitation of immigration status may trigger and dissuade some from further engaging in care, especially for undocumented immigrants.

While some experts encourage verbal discussion of how immigration status affects patients' health and access to care, they also support minimizing documentation due to the risks involved in the context of rapidly changing immigration policies with variable consequences to patients [12]. Proxy language to elicit immigration stressors includes listing a patient as "ineligible for insurance" and documentation of employment or family stressors. From an ethical perspective, the reporting of undocumented immigrants to officials contradicts the principle of nonmaleficence and has been denounced by the American Medical Association.

\section{Training Curricula}

The unique experiences of immigrants and immigrant communities must be taught and discussed in psychiatry residency curricula, and multiple resources exist for teaching about immigrant healthcare in the United States (Table 1). However, to relegate these topics to a "cultural seminar" that focuses on downloading information and statistics may risk further stereotyping and bias in considering the experiences of immigrants [13]. Rather, cultural and structural humility approaches to considering the mental health of immigrant populations should be incorporated into case conference discussions and didactics of various topics (e.g., neuropsychiatry, psychopharmacology, and forensic psychiatry). In the spirit of self-reflection, early trainees may benefit from an ethnographic approach where each trainee is called to reflect on the immigration experiences of their own families and the structural barriers faced by their ancestors (since, except for Indigenous people, all residents and citizens of the United States come from immigrant backgrounds) [14]. As trainees are able to see themselves as "situated" individuals with specific identities, experiences and histories, and "specific social and economic location that influences each and every interaction they have with patients [15]," they will hopefully be more able to identify their power and privilege with respect to the patient. The exploration of their own subjectivities and complexity helps them more effectively critique "objectivity" as it is valued in medicine and appreciate the depth and complexities of each individual patient. 
Table 1 Clinical, legal, and educational resources on immigrant healthcare

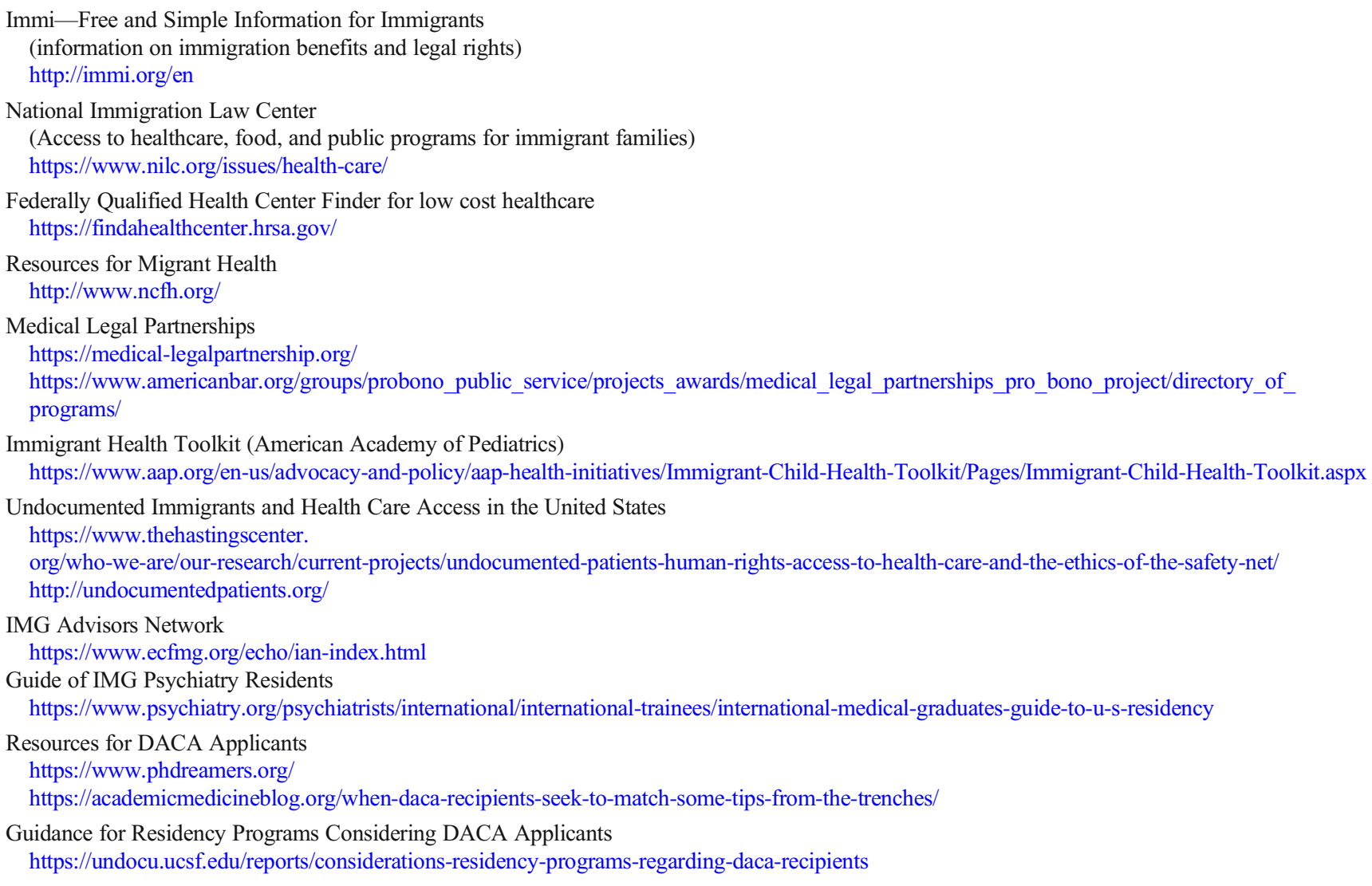

In acknowledging social, political, economic, and immigration factors that affect patient mental health, it must also be conceded that most psychiatry faculty do not possess the expertise to effectively educate trainees on issues affecting immigration and should therefore take an interprofessional and multidisciplinary approach to residency curriculum, including forming longitudinal relationships with immigrants' rights community groups, conducting site visits to immigrant health clinics, and compensating staff members from these groups to teach didactic sessions to residents within the psychiatric curriculum [13].

\section{Clinical Learning Environments}

For the most part, clinical learning environments are not equipped for learners to best develop their skills related to community and advocacy partnerships. As we learn more and more about the social determinants of health and mental health, we need to provide avenues for trainees to learn how to address them. In order to implement principles of cultural humility and structural competency, it is important for training programs to model and prioritize partnerships with local immigration experts and resource groups. This demonstrates that the educational program is committed to understanding the specific challenges of local immigrant communities and is supportive of efforts to achieve mental health equity for immigrant groups in their local contexts. At the same time, trainees learn community engagement and advocacy skills that can be applied to different locales throughout their careers.

A creative solution that is not only beneficial for training but directly benefits immigrant and underserved patients is medicallegal partnerships (MLPs). MLPs embed civil legal services attorneys into healthcare settings and on interprofessional healthcare teams delivering care to low income or otherwise vulnerable patients and communities [16]. MLPs present opportunities to instill in residents a practical understanding of social determinants of health and provide them with concrete tools for addressing them, as well as helping residents develop structural competence and build the necessary skills to address barriers to health at the patient, institutional, and population levels.

\section{Challenges Faced by Immigrant Trainees}

Reflecting on the value of patients and providers alike as "situated" individuals, two specific groups of immigrant students/ 
trainees-Deferred Action for Childhood Arrivals (DACA) recipients and international medical graduates (IMGs) - deserve special emphasis within the psychiatry graduate medical education setting. DACA recipients, with temporary protected status, work authorization, and access to health and social services are nevertheless subjected to the vicissitudes of the US immigration policy with the threat of having these privileges revoked, hence discouraging participation in prolonged educational and training programs like medicine. IMGs, in contrast, require visas and other special authorization to train and work in the US for discrete periods of time, often incurring large expenses to maintain legal employment status. This section will elucidate the specific challenges faced by these groups with respect to psychiatric training and discuss how training programs can better support trainees with immigration needs.

\section{DACA Recipients}

Medical students and resident/fellow trainees who are undocumented or have non-permanent resident/non-US citizen immigration status face unique hurdles while pursuing their training. Although there is generally a growing familiarity with some groups of immigrant categories, the fast-changing pace of immigration policy does not often allow medical school and graduate medical education program administrators to remain abreast of new developments that may challenge or facilitate training for these individuals. One growing group of immigrant medical students and residents are beneficiaries of the DACA program. The program, which began in 2012, offers relief from deportation to a subset of undocumented immigrants and makes them eligible for a social security number and work authorization. DACA recipients are currently able to renew their eligibility every two years; however, recent federal executive policies attempted to end the program in 2017, and the future of the program depends on judicial review of legal challenges regarding revocation of the program.

Since the inception of DACA, the Association of American Medical Colleges (AAMC) has reported increased numbers of medical student applicants identifying as having DACA status, although official numbers have not been published [17]. The AAMC maintains a yearly report identifying medical schools that accept applications from DACA beneficiaries. The 2020 report lists 65 schools that accept DACA applicants; however, each school enforces varied guidelines regarding the validity of a student's DACA status, or eligibility for financial aid [18]. DACA beneficiaries that successfully matriculate to medical school face further difficulties when entering the residency Match, as most residency programs have not verbalized their policies for consideration of DACA applicants. Furthermore, the uncertainty surrounding the US Supreme Court's pending decision prompted the National Resident
Matching Program (NRMP) to issue a statement addressing the potential end to the DACA program and confirming that the NRMP would grant waivers for programs and applicants that could not fulfill their match contract in the case of termination of the DACA program [19].

Although DACA has contributed to improvements in education opportunity, access to healthcare, social integration, and economic stability for beneficiaries of the program, DACA recipients have expressed ongoing stressors from their previous undocumented status, as well as from a sense of responsibility for family members that remain undocumented [20]. The temporary nature of the program also threatens the newfound stability for these students and trainees. Unfortunately, undocumented individuals that did not qualify for DACA status would be ineligible for employment upon completion of their medical education and most medical schools do not address whether they accept applications from undocumented students. Similarly, there is little information to guide students with unusual immigration documentation, such as individuals with temporary protected status (TPS) and others.

\section{IMGs}

Although IMGs consist of both US citizens and non-US citizens that complete their medical training outside of the US, our focus here is on non-US citizens only. IMGs are an important part of the American psychiatric workforce. They comprise about $30 \%$ of psychiatry trainees and $25 \%$ of the membership of the American Psychiatric Association [21]. IMGs face significant, long-term challenges and obstacles in obtaining residency positions in the US. IMGs who have completed post-graduate training in their home countries have to complete board requirements in the US to qualify to apply to US residency programs. Additional challenges include visa applications and maintaining legal presence status in the US for the trainee and their family members.

Most IMGs are admitted to the US for residency training on $\mathrm{J}-1$ visas sponsored by the Educational Commission for Foreign Medical Graduates (ECFMG), which certifies the educational credentials of IMGs. The maximum number of years one can stay in the US on a J-1 visa is 7 years, after which one is obligated to return to their country of origin for at least 2 years before being eligible to return to the US. Alternatively, the 2-year home residency requirement may be waived if the physician can document (1) persecution upon return to their home country, (2) residence would bring hardship to a spouse or children who are US citizens or permanent residents, or (3) employment with an Interested Governmental Agency, which includes medical service in Health Professional Shortage Areas (HPSAs), for 3 years. If IMGs are successful in obtaining a waiver, many choose to continue to live, work, and immigrate to the US. Following residency or fellowship, 
the H1-B non-immigrant visa provides more flexibility with a maximum duration of 6 years during which IMGs may apply for permanent residency through various routes, including employer sponsored labor certification [22, 23].

In recent years, hostile and unpredictable immigration policies have deterred IMGs from applying to residency programs with a decline of $10 \%$ of total applications in all specialties and $30 \%$ in psychiatry between 2015 and 2020 [24]. Similarly, the percentage of non-US citizen IMG physicians matching in psychiatry residency programs has declined significantly from a decade ago [22]. Immigration challenges deter GME programs from recruiting IMGs due to the potential for immigration issues affecting their legal presence, additional logistical requirements, extra immigration-related costs, and other complications. For example, many psychiatry trainees were affected by the Muslim Travel Ban of 2017 (Executive Order 13769), causing their training to be interrupted and affecting staffing [25]. Additional expansions of travel bans will have further consequences on the training of psychiatrists and the ability to meet the mental health needs of underserved and rural populations where many programs are IMG-dependent (i.e., more than $50 \%$ of residents are IMGs) [22].

\section{Additional Challenges for IMG Trainees}

Both citizen and non-citizen IMGs face significant differences in opportunities for success, starting with the decreased likelihood of getting into a residency program. This varies based on country of training and country of origin as well as ethnicity within each country [26]. Although program dependent, IMGs are often filtered out from residency applications, a convenient way of reducing the large volume of applications programs receive and action that reinforces longstanding structural discrimination against IMGs [27]. Self-identified challenges of IMG residents include dual learning curves of clinical medicine and cultural bias, global costs of leaving home countries, change in support systems, and career options after residency when visa issues resurface [5]. IMGs experience higher rates of discrimination in residency and as practicing physicians. In a national survey of residents, IMGs reported higher rates of racial and ethnic discrimination by patients, medical staff, medical students, and peers compared with US medical graduates [28].

In support of IMG psychiatrists, the APA has specific sections of its website dedicated to overviews of the US medical education training system, language and communication, cultural factors, and immigration system (Table 1). Overall, IMGs represent $26 \%$ of physicians in practice and $24 \%$ of residents in specialty programs [26]. IMG psychiatrists contribute significantly to the psychiatric workforce, especially in medically underserved and rural areas, and bring added perspectives to training, research, and curriculum through their own immigration and previous medical/psychiatric experiences [22, 23].

The discussion of acculturation of IMGs focuses particularly on the transition of those from "non-Western" cultures into American culture-deconstructed into such themes as individualism, personal expression of authority and status, egalitarianism, sexuality, gender roles, and the biopsychosocial model rather than the medical model of psychiatry [29]. Orientation programs and resources to prepare IMGs to address these topics should resist reverting to early cultural competence frameworks which risk stereotyping, erasing of intersectionality, and exertion of who is a "normal" or "typical" American, implicitly perpetuating IMGs as the foreign "other." In designing such material, a helpful approach would be to start with a needs assessment, as is typical of the process of developing any curriculum, while also explicitly and implicitly emphasizing the value of IMG trainees' experiences, histories, and narratives. Such orientations would be ripe opportunities to discuss cultural humility, intersectionality and to explore trainees situated selves as frameworks for critical thinking rather than emphasizing content about American life.

\section{Supporting DACA and IMG Trainees}

In support of DACA and IMG trainees, an approach toward holistic review of residency applications at all levels - screening, interviews, and ranking-reduces structural barriers and potentially discriminatory practices. Movement toward these goals include educational development of faculty and residents involved in recruitment in identifying their own biases (e.g., the belief that DACA/IMG applicants are less qualified) and recognizing the personal and sociopolitical challenges applicants have faced, sometimes referred to as a person's "distance traveled." Medical schools and residency programs should strive for transparency in their admissions policies regarding IMGs, DACA recipients, and undocumented students. Training programs should become equipped to better support trainees through the logistical challenges relating to their immigration status [30]. Given the large numbers of IMGs in the US physician workforce, programs can also provide intergenerational mentorship for IMGs with individuals who have institutional knowledge of navigating career and immigration challenges after residency (Table 1). Lastly, for trainees experiencing racial violence, which includes harassment and verbal assault, including from patients, a community and institutional response is required [31].

\section{Conclusion}

As the field of psychiatry struggles to meet the needs of an increasingly diverse patient population in increasingly 
divisive times in which immigration policies are rapidly changing, it is important to consider the mental health implications of immigration in medical students, residents, and subsequently, their patient populations, in order to effectively address deep-rooted mental health inequities. Immigrant trainees contribute significantly to the psychiatry workforce and bring a depth of perspective to training and educational experiences. Training programs and psychiatry departments must develop increasing understanding of the challenges and barriers that prevent the creation of an optimal educational and training environment for trainees who do not have permanent status or citizenship in the US. Faculty and supervisors must approach these challenges through a lens of cultural humility and self-reflection. Immigration is a social determinant of mental health that impacts all facets of the field of psychiatry, and its impacts are far-reaching.

Acknowledgments The authors would like to acknowledge Mallory Curran, JD, for her contributions to resources provided and for chairing a session that inspired some of the content of this manuscript, entitled "Immigration Status as a Social Determinant of Mental Health: What Can Psychiatrists Do to Support Patients and Communities? A Call to Action," presented at the American Psychiatric Association Annual Meeting on May 20, 2019 in San Francisco, CA.

\section{Compliance with ethical standards}

Disclosure On behalf of all authors, the corresponding author states that there is no conflict of interest.

\section{References}

1. World Health Organization. Closing the gap in a generation: health equity through action on the social determinants of health: Commission on Social Determinants of Health. 2008.

2. Paradies Y, Ben J, Denson N, Elias A, Priest N, Pieterse A, et al. Racism as a determinant of health: a systematic review and metaanalysis. PLoS One. 2015;10:e0138511.

3. Almeida J, Biello KB, Pedraza F, Wintner S, Viruell-Fuentes E. The association between anti-immigrant policies and perceived discrimination among Latinos in the US: a multilevel analysis. SSM Popul Health. 2016;2:897-903.

4. Gee GC, Ford CL. Structural racism and health inequities: old issues, new directions. Du Bois Rev. 2011;8:115-32.

5. Chen J, Vargas-Bustamante A. Estimating the effects of immigration status on mental health care utilizations in the United States. J Immigr Minor Health. 2011;13:671-80.

6. Finno-Velasquez M, Cardoso JB, Dettlaff AJ, Hurlburt MS. Effects of parent immigration status on mental health service use among Latino children referred to child welfare. Psychiatr Serv. 2016;67: 192-8.

7. Castañeda H, Holmes SM, Madrigal DS, Young M-ED, Beyeler N, Quesada J. Immigration as a social determinant of health. Annu Rev Public Health. 2015;36:375-92.

8. Fisher-Borne M, Cain JM, Martin SL. From mastery to accountability: cultural humility as an alternative to cultural competence. Soc Work Educ. 2015;34:165-81.

9. Yeager KA, Bauer-Wu S. Cultural humility: essential foundation for clinical researchers. Appl Nurs Res. 2013;26:251-6.
10. Tervalon M, Murray-García J. Cultural humility versus cultural competence: a critical distinction in defining physician training outcomes in multicultural education. J Health Care Poor Underserved. 1998;9:117-25.

11. Metzl JM, Hansen H. Structural competency: theorizing a new medical engagement withstigma and inequality. Soc Sci Med. 2014;103:126-33.

12. Kim G, Molina US, Saadi A. Should immigration status information be included in a patient's health record? AMA J Ethics. 2019;21:8-16.

13. Rao S, How PC, Ton H. Education, training, and recruitment of a diverse workforce in psychiatry. Psychiatr Ann. 2018;48:143-8.

14. Pryor A. Deep ethnography: culture at the core of curriculum. Language Arts. 2004;81:396-405.

15. Wear D. Insurgent multiculturalism: rethinking how and why we teach culture in medical education. Acad Med. 2003;78:549-54.

16. Paul EG, Curran M, Tobin TE. The medical-legal partnership approach to teaching social determinants of health and structural competency in residency programs. Acad Med. 2017;92:292-8.

17. Association of American Medical Colleges. Medical school policies on Deferred Action for Childhood Arrivals (DACA). 2018. Available from: https://aamc-orange.global.ssl.fastly.net/ production/media/filer_public/34/d9/34d992b9-50c0-4d20-958b8b8bcc761830/daca_policies_march_2018-.pdf. Accessed 2 Feb 2020.

18. Association of American Medical Colleges. Medical School Admission Requirements (MSAR) Advisor Report: Deferred Action for Childhood Arrivals (DACA). 2020. Available from: https://aamc-black.global.ssl.fastly.net/production/media/filer public/93/d5/93d5450d-1070-4fb5-8875-04c28f340db6/01msar_daca_policies_2020.pdf. Accessed 2 Feb 2020.

19. The National Resident Matching Program. NRMP Statement on Presidential Proclamation 9645 and Deferred Action for Childhood Arrivals (DACA). 2017. Available from: https:// mk0nrmp3oyqui6wqfm.kinstacdn.com/wp-content/uploads/2017/ 12/NRMP-Travel-Ban-and-DACA-Statement.pdf. Accessed 2 Feb 2020.

20. Sudhinaraset M, To TM, Ling I, Melo J, Chavarin J. The influence of deferred action for childhood arrivals on undocumented Asian and Pacific Islander young adults: through a social determinants of health lens. J Adolesc Health. 2017;60:741-6.

21. Majeed MH, Ali AA, Sudak DM. International Medical Graduates and American Psychiatry: The Past, Present, and Future. Acad Psychiatry. 2017;41:849-51.

22. Leon LR, Villar H, Leon CR, Psalms SB, Aranha G. The journey of a foreign-trained physician to a United States residency. J Am Coll Surg. 2007;204:486-94.

23. American Medical Association. Immigration information for international medical graduates. 2020. Available from: https://www. ama-assn.org/education/international-medical-education/ immigration-information-international-medical-graduates. Accessed 2 Feb 2020.

24. Association of American Medical Colleges. Medical Education: ERAS Statistics. 2020. Available from: https://www.aamc.org/ eras-statistics-2019. Accessed 2 Feb 2020.

25. Walaszek A. American Association of Directors of Psychiatric Residency Training (AADPRT) Position Statement on the Executive Order on Immigration. Acad Psychiatry. 2017;41:292.

26. Ranasinghe PD. International Medical Graduates in the US Physician Workforce. J Am Osteopath Assoc. 2015;115:236-41.

27. Desbiens NA, Vidaillet HJ. Discrimination against international medical graduates in the United States residency program selection process. BMC Med Educ. 2010;10:5-10.

28. Coombs AAT, King RK. Workplace discrimination: experiences of practicing physicians. J Natl Med Assoc. 2005;97:467-77. 
29. American Psychiatric Association. Navigating psychiatry residency in the United States: a guide for international medical graduate physicians. 2018. Available from: https://www.psychiatry.org/ psychiatrists/international/international-trainees/internationalmedical-graduates-guide-to-u-s-residency/cultural-factors. Accessed 2 Feb 2020.

30. Nakae S, Marquez DR, Di Bartolo IM, Rodriguez R. Considerations for residency programs regarding accepting undocumented students who are DACA recipients. Acad Med. 2017;92:1549-54.

31. Williams JC, Rohrbaugh RM. Confronting racial violence: resident, unit, and institutional responses. Acad Med. 2019;94:1084-8.

Publisher's Note Springer Nature remains neutral with regard to jurisdictional claims in published maps and institutional affiliations. 